\title{
Oncological management of advanced neuroendocrine tumours (Review)
}

\author{
PAWEŁ GUT \\ Department of Endocrinology, Metabolism and Internal Diseases, \\ Poznan University of Medical Sciences, Poznań 60-355, Poland
}

Received February 7, 2020; Accepted June 2, 2020

DOI: $10.3892 / \mathrm{mco} .2020 .2078$

\begin{abstract}
The oncological principles of managing patients with gastroenteropancreatic neuroendocrine tumours (GEP-NETs) depends on a number of factors and requires a multidisciplinary approach. Recent data have provided additional therapeutic options, including biotherapy, traditional chemotherapy and novel targeted agents. Somatostatin analogues (SSAs) inhibit multiple cellular functions, including secretion, motility and proliferation. Interferon appears to act through several mechanisms, with antisecretory effects, immunomodulatory effects and antiproliferative functions, the latter inhibiting direct growth or attenuating angiogenesis. Opinions on when to commence chemotherapy for well differentiated GEP-NETs varies among experts. In previous years, reserving chemotherapy for patients with progressive disease (well differentiated, inoperable and/or metastatic GEP-NETs) was reasonably well argued for. Most well differentiated endocrine tumours are richly vascular and many express vascular endothelial growth factor (VEGF) receptors. In a xenograft model of a human carcinoid, treatment with an anti-VEGF monoclonal antibody was revealed to inhibit tumour growth and metastasis. As the role of angiogenesis and hypoxic-associated factors appears to be associated with tumour aggressiveness, strategies using agents which target angiogenesis have been developed. Mammalian target of rapamycin (mTOR) is a conserved serine-threonine kinase that regulates the cell cycle and metabolism in response to environmental factors. In addition, mTOR inhibition suppression was demonstrated to suppress NET growth._Each patient requires an individual approach to the choice of therapy, which should be selected depending on the severity of disease.
\end{abstract}

Correspondence to: Dr Paweł Gut, Department of Endocrinology, Metabolism and Internal Diseases, Poznan University of Medical Sciences, 49 Przybyszewski Street, Poznań 60-355, Poland

E-mail: gutpj@poczta.onet.pl

Key words: oncological management, neuroendocrine tumour

\author{
Contents \\ 1. Introduction \\ 2. Biotherapy \\ 3. Interferon \\ 4. Chemotherapy \\ 5. Chemotherapy for well differentiated digestive GEP-NETs \\ 6. Targeted therapies in advanced GEP-NETs \\ 7. Conclusions
}

\section{Introduction}

The oncological principles of management in patients with gastroenteropancreatic neuroendocrine tumours (GEP-NETs) depend on a number of factors and require a multidisciplinary approach. Curative surgery is rarely possible in patients with metastatic disease, and other approaches are therefore necessary. Antiproliferative treatment decisions depends on a number of key factors: Firstly, the origin of the primary tumour; secondly, the histological differentiation and tumour grade, and finally on the aggressiveness and proliferative capacity of the tumour. Unlike other solid tumours in the digestive tract, wait-and-see strategies can often be adopted in GEP-NET patients. It is associated with the highly differentiated nature of neuroendocrine tumors and the often slow progression of the disease. Recent data have changed the therapeutic options and the results of biotherapy, traditional chemotherapy and new targeted agents have opened an exciting volley of therapies in this ever changing field. Well-coordinated international multicentre trails have afforded the opportunity of pooling resources in a field of rare tumour disease and to respond to interesting clinical questions.

\section{Biotherapy}

Somatostatin analogue treatment in GEP-NETs. Somatostatin and its analogues (SSAs) inhibit multiple cellular functions, including secretion, motility and proliferation. Its action is mediated by five specific somatostatin receptors (sstr 1-5), which belong to the $\mathrm{G}$ protein-coupled receptor family. The five receptors bind the natural peptide with high affinity, but only sstr2, sstr3 and sstr5 bind the short synthetic analogues used to treat GEP-NET patients. SSAs have been used 
1 successfully to treat functional GEP-NETs (e.g. carcinoid syndrome, VIP-omas) (1). The basis for the use of SSAs is the expression of somatostatin receptor subtypes in $80-90 \%$ of GEP-NETs according to autoradiographic or scintigraphic studies (2). The biological effects of SSAs occur in relation to receptor subtype interaction; inhibition of secretion appears to be largely mediated via the effects of the sstr2 subtype, and all commercially available SSAs have appreciable affinity for sstr2 $(3,4)$. However, proliferation in endocrine tissue may be mediated via other receptor subtypes (5).

Anti-tumour effect of SSAs. The initial studies examining the antiproliferative role of SSAs are difficult to interpret due to: The heterogeneity of tumour types (site of origin, tumour histology, tumour load and type of metastatic disease); the use of different formulations and doses; the lack of objective tumour progression prior to treatment with SSAs, meaning that disease stabilisation may also be attributable to the slowly progressive natural history common to many GET-NETs, and until recently, the complete lack of any randomised study examining this question $(6,7)$. Eriksson and Oberg collected data from 62 published studies or mini-series pertaining to treatment with octreotide: Tumour shrinkage was reported in only $10-20 \%$ of patients, but stabilisation of tumour growth for 8-16 months could be achieved in about half of the patients (8). Other data, mostly examining the efficacy of long-acting forms of SSAs, suggest that, overall, objective responses are rare $(<10 \%)$ with reasonable disease stabilisation in up to $50 \%$ of patients (with a response duration of 10-25 months) (9). A phase III study comparing lanreotide with interferon, and their combination, showed a partial tumour response of only $4 \%$ for the lanreotide arm, whereas disease stabilisation was observed in $28 \%$ of patients (10). Disease stabilisation thereafter confirmed in two further reports, with rates of 46 and $16 \%$ for octreotide doses of 450 and $600 \mathrm{mg} /$ day, respectively (11). Results appear to be similar for both functioning and non-functioning tumours (12). The publication of a randomized trial comparing Sandostatin-LAR $30 \mathrm{mg}$ every 28 days to placebo provided a definitive answer to the antiproliferative effects of SSAs in patients with metastatic midgut disease (13). In third important trial, 85 patients were randomized to receive $30 \mathrm{mg}$ Sandostatin-LAR or placebo. The median time to progression was significantly longer in the octreotide arm (14.3 months vs. 6 months) and the overall reduction in risk of tumour progression attributable to Sandostatin-LAR was $66 \%$ (HR 0.34). This effect was achieved via disease stabilization; there were no complete responses and only 1 partial response in treatment and placebo arms, respectively. The antiproliferative effect was visible in patients with or without carcinoid syndrome. At subanalysis, the most favourable outcomes were observed in patients with a small $(<10 \%)$ hepatic tumour loadband in those whose primary tumour had been resected. These data broadly confirm that SSAs have a real antiproliferative effect and that they should be considered in patients with advanced carcinoid tumours. The published CLARINET study results, confirmed the antiproliferative effect of lanreotide. The study was participated by 204 patients with NEN G1 and G2 (Ki $67<10 \%$ ), non-functioning with primary site in pancreas (45\%), midgut (36\%), hindgut (7\%) and unknown (13\%) (14). The development of agents such as pasireotide, capable an if increased affinity to certain sstr subtypes (e.g., bi-specific sstr2 and sstr5, or multi-specific binding capacity), or chimeras capable of recognising both sstr2 and dopamine D2 receptors (D2 was also recently identified in GEP-NETs) (15). May prove more efficacious in antiproliferative terms. Indeed, the dopamine-somatostatin chimeric molecule, BIM-23A760, has been shown to be efficacious in the control of cell growth from primary cultures of human non-functioning pituitary adenomas in a multi-centre study (16). However, use of such agents appears to be cell specific as highlighted by a recent in vitro analysis (17).

\section{Interferon}

Interferon appears to act through several mechanisms, with antisecretory, immunomodulatory effects, and antiproliferative functions (18), the latter in relation to direct growth inhibition or the attenuation of angiogenesis. Data interpretation is hampered by the use of varying types of interferon (INF- $\alpha-2 \alpha$, INF- $\alpha-2 \beta$ and human leukocyte interferon HuINF- $\alpha$-Le) combined with non-randomised heterogeneous studies in relatively small numbers of patients. Similarly to SSAs, the use of interferon in GEP-NETs with carcinoid syndrome has been found to be beneficial in controlling symptoms $(60 \%$ reduction in flushes and diarrhoea, respectively) and biochemical tumour markers. However, objective tumour responses are rare. Disease stabilisation, with standard doses of 3-9 MIU three times weekly, is reported to occur in approximately $35 \%$ of patients, with a median duration of response of 32 months (19). Higher doses do not confer a therapeutic advantage. In a recent phase III trial 64 patients with documented progressive, unresectable, metastatic carcinoid tumours ( $>60 \%$ were midgut in origin) were randomized between 5-fluorouracil and streptozocin (day 1-5) and recombinant INF- $\alpha-2 \alpha$ (3MU, 3 per week) (20). The median PFS for chemotherapy was 5.5 months vs. 14.1 for IFN [HR: 0.75 (0.41-1.36)]. Overall survival, tolerance, and effects on carcinoid symptoms were not significantly different. A long-acting preparation (pegylated interferon, PEG-INF), which achieves constant plasma concentrations with fewer adverse events 100 (which can include flu-like symptoms, fatigue, haematological 101 toxicity, etc.) was recently compared to bevacizumab in a 102 phase II trial, in patients with metastatic or unresectable 103 carcinoid disease (21). Forty-four patients on stable doses 104 of octreotide were randomly assigned to 18 weeks of beva- 105 cizumab or PEG-INF- $\alpha-2 \beta$. The results of PEG-INF- $\alpha-2 \beta 106$ compared to bevacizumab respectively were as follows: 0 vs. 107 $4(18 \%)$ partial responses, $15(68 \%)$ vs. 17 (77\%) stable disease, 108 and $6(27 \%)$ vs. $1(5 \%)$ progressive disease. The PFS rate after 109 18 weeks was $95 \%$ in bevacizumab vs. $68 \%$ on the PEG INF 110 arm (22). Overall, the interferon was well tolerated. A major 111 limitation of this study was the lack of documented disease 112 progression in all patients randomized, thus results pertaining 113 to disease stabilization could not be interpreted.

\section{Chemotherapy}

General principals. Opinions on when to commence chemo- 118 therapy for well differentiated GEP-NETs varies among 119 experts. In years past, reserving chemotherapy for patients 120 
with progressive disease (well differentiated, inoperable, and/or metastatic GEP-NETs) was reasonably well argued for. The slow natural progression of GEP-NETs in many patients allows for careful monitoring and the instigation of treatment once disease progression is documented. The definition of a well differentiated tumour will no doubt require clarification, as we have seen recently there is a difference between natural history and response to therapy according to tumour grade (above or below Ki-67 at 3\%) (23). Is it therefore reasonable to adopt a wait-and-see attitude for patients with well differentiated grade 2 tumours $(\mathrm{Ki}-67>3 \%)$. The anti-proliferative effects of SSAs would appear to be most applicable to grade 1 tumours, and the use of SSAs monotherapy (at least in GEP-NETs of midgut origin) in this setting may be reasonable, which reserves chemotherapeutic agents for documented progressive disease. Most experts would argue for early chemotherapy in patients with well-differentiated bulky disease at the outset or poorly differentiated tumours. Accurate histological classification is not always easy, as interobserver differences among pathologists are not uncommon (24). Guidelines to increase uniformity in this respect are required. The importance of accurate histology cannot be underestimated, and in cases where doubt exists, slides should be re-examined by several independent histopathologists. The use of the Ki-67 proliferation index has been helpful in distinguishing certain tumours and guiding treatment regiments. This marker is invariably high $(>20 \%)$ in poorly differentiated lesions and identification of grade 3 GEP-NETs is usually reasonably simple. However, it may be difficult to choose appropriate therapy in cases where the histological architecture resembles a well differentiated tumour but there is a moderately elevated Ki-67 (3-20\%) or borderline tumours, indeed, the gap between 3 and $20 \%$ may be too generous. Analysing biopsy samples, compared to larger operative specimens, poses special problems in performing estimates (25). The appraisal of proliferation indices and their relationship to treatment outcomes is required in future study protocols. Apart from histological differentiation, the type of chemotherapy has been largely based on the site of origin of the primary tumour. To date, this paradigm remains pertinent but may change in the future with the discovery of agents, cytotoxics or targeted therapies that are universally applicable to GEP-NETs.

New molecular predictors. Recent efforts have been made to try to determine molecular predictors of response to therapy. O-6-methylguanine-DNA methyltransferase (MGMT) deficiency, measured by immunohistochemistry, was found to predict a better response to temozolomide-based therapy (26). A further study in a group of 60 GEP-NET patients treated with chemotherapeutic agents a number or markers were found to be associated with response to individual therapies (including tyrosine kinase, Akt, thymidylate synthase (TS), phosphatase and tensin homologue (PTEN), Ki-67 and the hypoxic factor CA9 (27). These results demonstrate a number of new prognostic biomarkers in GEP-NETs, and in addition, response to chemotherapy was correlated with a simple panel of selected markers [such as CA9, Akt, PTEN, TS, and mismatch repair gene-human mutL homologue 1 (hLMH1)]. Tailoring therapies to suit individual patients should become possible as the molecular events associated with treatment responses are revealed.

\section{Chemotherapy for well differentiated digestive GEP-NETs}

\section{1}

Pancreatic. Apart from insulinomas, other pancreatic GEP-NETs are frequently associated with metastatic disease and curative surgical options are rarely $(<25 \%)$ possible $(28)$. Single-agent chemotherapy with streptozocin yielded tumour response rates of $36-42 \%$, but these early studies can be criticised for using crude methods to interpret morphological responses (29). Other monotherapies, including chlorotozotocin, doxorubicin, 5-fluorouracil (5-FU) and dacarbazine have been used, but criticised either for a high toxicity rate or a lack of objective response. Streptozocin, initially combined with 5-FU, and subsequently with doxorubicin yielded a $69 \%$ objective response rate and a median survival of 26 months, in a study by Moertel et $a l$ and this combination became the standard therapy (30). While no group has managed to achieve the same response rates as Moertel, objective responses of 36-55\% have been established using streptozocin and doxorubicin, with the exception of two small retrospective studies where response rates of $6 \%$ were reported in both studies (31). However, in a more recent study by Delaunoid et al, 45 patients showed a $36 \%$ overall response rate using well-defined criteria for recruitment and evaluation (32), in addition, the 2- and 3 -year overall survival rates were 50 and $24 \%$, respectively. Such discrepancies are likely to be related to differences in overall study size and differences in the criteria used to measure response. The triple combination streptozocin, doxorubicin and 5-FU gave a 39\% objective response, and more recently, streptozocin and liposomal doxorubicin a $40 \%$ response rate (33). This latter study was interesting in that it reported no cardio-toxicity (the prevalence of cardiomyopathy increases significantly when patients are given doses of doxorubicin $>550 \mathrm{mg} / \mathrm{m}^{2}$ ). The use of streptozocin requires careful monitoring of renal function. Other combinations used have included capecitabine and oxaliplatin with a $27 \%$ response rate and stabilization achieved in $45 \%$ of patients (34). Gemcitabine and oxaliplatin give a $40 \%$ objective response in a small number of patients (35). Temozolomide, an oral form of dacarbaxine, had recently gained favour in the treatment of pancreatic GEP-NETs. Dacarbaxine monotherapy (i.v. 100 every 4 weeks) was previously shown to give 39\% response 101 rates (36), and the interesting results achieved by temozolo- 102 mide (37) in central nervous system tumours prompted its use 103 in GEP-NETs. Although the data surrounding temozolomide 104 remain relatively preliminary, 8-70\% response rates have been 105 achieved either as monotherapy or in combination with thalid- 106 omide or capecitabine (38). Temozolomide has the advantage 107 of being relatively well tolerated, being available as an oral 108 therapy and has been reported to give impressive disease 109 stabilization in heavily pre-treated patients. A direct random- 110 ized comparison of temozolomide alone or in combination 111 with other therapies, or of temozolomide with either standard 112 chemotherapy (streptozocin and doxorubicin) or targeted 113 therapies (sunitinib or everolimus) would appear to be logical 114 steps in testing this interesting molecule.

Midgut. Multiple molecules, used as either a single-agent or 117 in combination strategies, have been tested to treat gastroin- 118 testinal and largely midgut NETs with disappointing objective 119 response rates ranging from 0 to $40 \%$ and response durations 120 
rarely exceeding 3 months (39). Again, a 5-FU and streptozocin combination initially gave a $33 \%$ response rate but later studies using the same combination were disappointing (40). More recent studies have failed to justify cytotoxic combinations in midgut GEP-NETs. A large phase II/III trial evaluated 176 patients randomized to streptozocin plus 5-FU or doxorubicin plus 5-FU (41). Patients crossed over to dacarbazine treatment after disease progression following first-line treatment. There were no differences in response rate (16 and $15.9 \%$, respectively) and PFS was 5.3 and 4.5 months respectively in both arms. However, patients randomized to streptozocin plus 5-FU experienced a longer survival (24.3 months) than the patients randomized to doxorubicin plus 5-FU (15.7 months). The response rate of crossover dacarbazine treatment was $8.2 \%$, with a median survival of 11.9 months. Recent trials examining oxaliplatin in combination with either gemcitabine or capcetabine report no response rates. Temozolomide has yielded modest response rates in treating advanced midgut GEP-NETs (9\%) but this single agent achieved respectable stabilization (70\%) in patients heavily pre-treated prior to temozolomide commencement. Overall, international recommendations have suggested abandoning the use of classical cytotoxics in the treatment of metastatic midgut tumours in favour of more suitable options when applicable (e.g., transarterial chemoembolization, peptide receptor radionucleotide therapy (PRRT), targeted agents, or enrolment in clinical trials) (42).

\section{Targeted therapies in advanced GEP-NETs}

Bevacizumab. Most well differentiated endocrine tumours are richly vascular and many express VEGF receptors (43). In a xenograft model of a human carcinoid, treatment with an anti-VEGF monoclonal antibody was found to inhibit tumour growth and metastasis (44). As the role of angiogenesis and hypoxic-related factors appears to be clearly related to tumour aggressiveness, strategies using agents which target angiogenesis have been developed. Bevacizumab with depot octreotide gave a partial response of $18 \%$ and a $77 \%$ disease stabilization in a recent phase II trial (45). In this trial, bevacizumab was also demonstrated to inhibit tumour blood flow at day 2 and week 18. Bevacizumab in combination with chemotherapy is also under examination. Preliminary results of a phase II trial combining bevacizumab, capecitabine plus oxaliplatin in 40 patients with advanced disease (20 pancreas, 5 small bowel and 15 unknown or other GEP-NETs) reported 7 partial responses (23\%; 6 had pancreatic primaries) with a median PFS of 13.7 months (46). Another preliminary report from a phase II study examining bevacizumab and Folfox in non-pancreatic GEP-NETs showed 20\% (1/5) partial responses and $80 \%(4 / 5)$ stabilizations and slightly poorer results for pancreatic GEP-NETs (partial responses 33\% (2/6) and $66 \%(4 / 6)$ stabilizations (47).

Tyrosine kinase inhibitors. A number of small molecule tyrosine kinase inhibitors have been evaluated in advanced NETs. The most promising activity has been observed with sorafenib, pazopanib, or sunitinib, all of which have activity against VEGF receptor (VEGFR). The small molecule tyrosine kinase inhibitor sorafenib has activity against VEGFR-2 and platelet-derived growth factor receptor B (PDGFR-B), and was evaluated in 50 patients with carcinoid tumours and 61 43 patients with pancreatic NETs. In a preliminary analysis, 62 responses were observed in 7\% of the carcinoid patients and 63 $11 \%$ of the patients with pancreatic NETs (48). Pazopanib, a 64 tyrosine kinase inhibitor of VEGFR-1, VEGFR-2, VEGFR-3, PDGFR- $\alpha / \beta$, and c-kit, was evaluated in a prospective study of 51 patients with advanced NETs (29 with pancreatic NETs and 22 with carcinoid tumours) on stable doses of octreotide-LAR. Patients received pazopanib at a dose of $800 \mathrm{mg}$ daily. The response rate among patients with pancreatic NETs was 17\%; no patients with carcinoid experienced a radiographic response (by response evaluation criteria in solid tumours-RECIST) (49). Sunitinib works by blocking multiple molecular targets implicated in the growth, proliferation and metastatic spreading, of tumour cells acting via VEGF-R, PDGFR and other targets important to tumour growth, including KIT, FLT3 and RET (50). The first report of clinical activity for sunitinib in GEP-NETs was recently reported by Kulke et al (51). This was a phase II trial involving 107 patients with mixed GEP-NETs treated with sunitinib (carcinoid tumours, $n=41$; pancreatic NETs, $n=66$ ). Respective overall objective response rate in pancreatic and carcinoid GEP-NETs were 17\% (11/66) and 2\% (1/41); the corresponding disease stabilization rates for both tumour groups were 68\% (45/66) and 83\% (34/41), respectively. The median time to tumour progression was 7.7 months in pancreatic GEP-NETs patients and 10.2 months in carcinoid tumour patients. One-year survival rate was $>80 \%$ in both groups of patients. The treatment was well tolerated. A recent phase III trial examining sunitinib as monotherapy vs. placebo in 340 planned patients was performed in well differentiated advanced and progressive pancreatic GEP-NETs. Prior systemic therapy had been administered to 66 and $72 \%$ of patients treated in the treatment vs. placebo arms, respectively. The study was terminated early at an unplanned interim analysis after enrolment of 171 patients and 81 PFS events. The median PFS (primary endpoint) was 11.4 months in the sunitinib arm vs. 5.5 months in the placebo arm (HR 0.418). However, due to the number of interim looks, the PFS difference did not reach statistical significance. Objective response rates for sunitinib was 9.3 vs. $0 \%$ for placebo and objective progression was almost double in the placebo arm (27.1 vs. $14.0 \%)$. These results 10 were achieved at the expense of non-negligible toxicity for the 102 active treatment arm (notably diarrhoea (59 vs. 38\%), hand-foot 103 syndrome (22.9 vs. $2.4 \%$ ) and hypertension (26.5 vs. $4.9 \%)$ (52). 104

mTOR inhibitors. Mammalian target of rapamycin (mTOR) is a 106 conserved serine-threonine kinase that regulates the cell cycle 107 and metabolism in response to environmental factors. It mediates 108 signalling transduction downstream of receptor tyrosine kinases 109 and has been linked to pathways involved in the pathogenesis 110 of GEP-NETs in several models. In addition, mTOR inhibition 111 suppression was found to suppress NET growth (53). Yao et al 112 conducted an initial phase II study using the mTOR inhibitor 113 RAD001 (everolimus), at two does (5 or $10 \mathrm{mg} /$ day) in associa- 114 tion with octreotide LAR $30 \mathrm{mg}$ every 28 days in a group of 115 30 patients with pancreatic GEP-NETs and 30 with non-pancre- 116 atic gastrointestinal GEP-NETs (54). The intent-to-treat 117 response rate was $20 \%$ and per protocol, there were 13 patients 118 with partial responses (PR; 22\%), 42 with stabile disease 119 (SD; 70\%), and five patients with progressive disease (PD; 8\%). 120 
Overall median PFS was 60 weeks. Median overall survival had not been reached and 1-, 2- and 3-year survival rates were 83,81 , and $78 \%$, respectively. Mild aphthous ulceration occurred in $8 \%$ of patients and the grade 3/4 toxicities occurring in $>10 \%$ of patients included hypophosphatemia (11\%), fatigue (11\%), and diarrhoea (11\%). Importantly, anti-tumour activity was noted for patients with non-pancreatic gastrointestinal GEP-NETs: 5/30 (17\%) confirmed partial responses, 24 SD (80\%), and one PD (3\%). In the pancreatic GEP-NETs group, there were 8/30 PR (27\%), 18 SD (60\%), and 4 PD (13\%). Response was higher for the $10 \mathrm{mg}$ RAD001 dose cohort (30 vs. 13\% PR). These results paved the way for a large open-labelled phase II trial (Radiant 1), which examined, in a stratified manner, everolimus (RAD001) $10 \mathrm{mg} /$ day and everolimus $10 \mathrm{mg}$ /day plus octreotide depot (every 28 days) in patients with advanced pancreatic GEP-NETs with progression during or after chemotherapy. Synergy between RAD001 and octreotide had been previously suggested as octreotide may protect against a potential RAD001 resistance mechanism via inhibition of IGF pathways (55). Radiant I demonstrated a PR of $9.6 \%$ for everolimus and $4.4 \%$ for everolimus/octreotide therapy. The corresponding rates of disease stabilizations were 68 and $80 \%$ for the monotherapy and combined therapy groups, respectively; median PFS was 9.7 months for everolimus alone and 17.7 months for everolimus combined with octreotide. Thereafter two large randomized phase III trials examined the use of everolimus in the treatment of pancreatic and non-pancreatic NETs. Radiant III compared everolimus $10 \mathrm{mg} /$ day alone to placebo and best supportive care in 410 pancreatic NET patients (56). The groups were well matched for disease extent and prior therapies before study enrolment. The primary endpoint, PFS, was significantly longer in the everolimus group (11.0 months vs. 4.6 months; HR 0.35). While confirmed responses were only $4.8 \%$ in the active arm (vs. $2.0 \%$ in placebo), overall disease control rates (complete or partial response and SD) was significantly higher in the everolimus arm (78 vs. 53\%). Radiant II was a similar study in a more mixed group of patients with advanced NETs and carcinoid syndrome $(n=429)$ where everolimus with octreotide was compared with placebo/octreotide combinations (57). The primary endpoint, PFS, almost achieved statistically significance (16.4 months vs. 11.3 months in everolimus vs. placebo groups; HR 0.77). Imbalances in the groups were noted as the everolimus/octreotide group had significantly more lung NET primaries $(15$ vs. $5 \%, \mathrm{P}<0.05)$ and had received more systemic chemotherapy ( 35 vs. $26 \%, \mathrm{P}<0.05$ ) than the placebo/octreotide group (58).

\section{Conclusions}

Approximately two-thirds of malignant GEP-NETs are metastatic at discover. Surgery is possible in only a minority of patients, and therefore chemotherapy, with or without other strategies (e.g. local ablation), is frequently indicated in patients with symptomatic, bulky or progressive disease (59). For well-differentiated pancreatic GEP-NETs the reference association of streptozocin/doxorubicin (or 5-FU) yields objective responses in approximately $35-40 \%$ of patients but treatment is limited due to the potential toxicity. The approval of sunitinib in advanced progressive pancreatic GEP-NETs allows for a welcome alternative therapy and while responses rates are low, disease stabilizations appear 61 impressive. Similarly, everolimus will almost certainly be 62 approved for the same indication, allowing for a number of strategies to be employed in cases of advanced pancreatic GEP-NETs. Temozolomide appears to have impressive anti-tumour activity for pancreatic GEP-NETs and requires comparison with other established therapies. Published studies which evaluate chemotherapy for midgut and other gastrointestinal GEP-NETs are poor, outdated, disappointing, and cannot be recommended. In patients with low-volume and grade 1 tumours, somatostatin analogues are effective in preventing disease progression. The results of everolimus in combination with octreotide are formally awaited and may prove an alternative strategy. However, therapies such as chemoembolization or peptide receptor radionuclide therapy should be considered in gastrointestinal GEP-NETs. Little progress has been made for poorly differentiated GEP-NETs that respond to platinum/etoposide combined therapies but where disease control proves to be limited $(60,61)$.

\section{Acknowledgements}

Not applicable.

\section{Funding}

No funding was received.

\section{Availability of data and materials}

The datasets used and/or analysed are available from the corresponding author on reasonable request.

\section{Authors' contributions}

PG designed the current review, wrote the manuscript, edited and analysed the data.

\section{Ethics approval and consent to participate}

Not applicable.

Patient consent for publication

Not applicable.

Competing interests

The author declares that they have no competing interests.

\section{References}

1. Modlin IM, Pavel M, Kidd M and Gustafsson BI: Review article 113 somatostatin analogues in the treatment of gastroenteropan- 114 creatic neuroendocrine (carcinoid) tumours. Aliment Pharmacol 115 Ther 31: 169-188, 2010.

2. Krenning EP, Kwekkeboom DJ, Bakker WH, Breeman WA, 116 Kooij PP, Oei HY, van Hagen M, Postema PT, de Jong M 117 and Reubi JC, et al: Somatostatin receptor scintigraphy with 118 [111In-DTPA-D-Phe1]- and [1231-Tyr3]-octreotide: The Rotterdam experience with more than 1000 patients. Eur J Nucl Med 20: 716-731, 1993. 62 63 64 
3. Nilsson O, Kolby L, Wangberg B, Wigander A, Billig $\mathrm{H}$ William-Olsson L, Fjälling M, Forssell-Aronsson E and Ahlman H: Comparative studies on the expression of somatostatin receptor subtypes, outcome of octreotide scintigraphy and response to octreotide treatment in patients with carcinoid tumours. Br J Cancer 77: 632-637, 1998.

4. Bruns C, Lewis I, Briner U, Meno-Tetang G and Weckbecker G: SOM230: A novel somatostatin peptidomimetic with broad somatotropin release inhibiting factor (SRIF) receptor binding and a unique antisecretory profile. Eur J Endocrinol 146: 707-716, 2002.

5. Reubi JC, Kvols LK, Waser B, Nagorney DM, Heitz PU, Charboneau JW, Reading CC and Moertel C: Detection of somatostatin receptors in surgical and percutaneous needle biopsy samples of carcinoids and islet cell carcinomas. Cancer Res 50 5969-5977, 1990.

6. Aparicio T, Ducreux M, Baudin E, Sabourin JC, De Baere T, Mitry E, Schlumberger M and Rougier P: Antitumour activity of somatostatin analogues in progressive metastatic neuroendocrine tumours. Eur J Cancer 37: 1014-1019, 2001.

7. Ducreux M, Ruszniewski P, Chayvialle JA, Blumberg J, Cloarec D, Michel H, Raymond JM, Dupas JL, Gouerou H, Jian R, et al: The antitumoral effect of the long-acting somatostatin analog lanreotide in neuroendocrine tumors. Am J Gastroenterol 95: 3276-3281, 2000.

8. Eriksson B and Oberg K: Summing up 15 years of somatostatin analog therapy in neuroendocrine tumours: Future outlook. Ann Oncol 10 (Suppl 2): S31-S38, 1999.

9. Ricci S, Antonuzzo A, Galli L, Ferdeghini M, Bodei L, Orlandini C and Conte PF: Octreotide acetate long-acting release in patients with metastatic neuroendocrine tumours pretreated with lanreotide. Ann Oncol 11: 1127-1130, 2000.

10. Kolby L, Persson G, Franzen S and Ahrén B: Randomized clinical trial of the effect of interferon alpha on survival in patients with disseminated midgut carcinoid tumours. Br J Surg 90: 687-693, 2003.

11. Faiss S, Pape UF, Bohmig M, Dörffel Y, Mansmann U, Golder W, Riecken EO and Wiedenmann B; International Lanreotide and Interferon Alfa Study Group: Prospective, randomized, multicentre trial on the antiproliferative effect of lanreotide, interferon alfa, and their combination for therapy of metastatic neuroendocrine gastroenteropancreatic tumors-the International Lanroetid and Interferon Alfa Study Group. J Clin Oncol 21: 2689-2696, 2003.

12. Arnold R, Rinke A, Klose KJ, Müller HH, Wied M, Zamzow K, Schmidt C, Schade-Brittinger C, Barth P, Moll R, et al: Octreotide versus octreotide plus interferon-alpha in endocrine gastroenteropancreatic tumors: A randomized trial. Clin Gastroenterol Hepatol 3: 761-771, 2005.

13. Rinke A, Muller HH, Schade-Brittinger C, Klose KJ, Barth P, Wied M, Mayer C, Aminossadati B, Pape UF, Bläker M, et al: Placebo-controlled, double-blind, prospective, randomized study on the effect of octreotide LAR in the control of tumour growth in patients with metastatic neuroendocrine midgut tumours: A report from the PROMID Study Group. J Clin Oncol 27: 4656-4663, 2009.

14. Caplin ME, Pavel M, Ćwikła JB, Phan AT, Raderer M, Sedláčková E, Cadiot G, Wolin EM, Capdevila J, Wall L, et al: Anti-tumour effects of lanreotide for pancreatic and intestinal neuroendocrine tumours: The CLARINET open-label extension study. Endocr Relat Cancer 23: 191-199, 2016.

15. O'Toole D, Savenau A, Couvelard A, Gunz G, Enjalbert A, Jaquet P, Ruszniewski P and Barlier A: The analysis of quantitative expression of somatostatin and dopamine receptors in gastro-entero-pancreatic tumours opens new therapeutic strategies. Eur J Endocrinol 155: 849-857, 2006

16. Florio T, Barbieri F, Spaziante R, Zona G, Hofland LJ, van Koetsveld PM, Feelders RA, Stalla GK, Theodoropoulou M, Culler MD, et al: Efficacy of a dopamine-somatostatin chimeric molecule, BIM-23A760, in the control of cell growth from primary cultures of human non-functioning pituitary adenomas: A multi-center study. Endocr Relat Cancer 15: 583-596, 2008.

17. Kidd M, Drozdov I, Joseph R, Pfragner R, Culler M and Modlin I: Differential cytotoxicity of novel somatostatin and dopamine chimeric compounds on bronchopulmonary and small intestinal neuroendocrine tumor cell lines. Cancer 113: 690-700, 2008

18. Shah T and Caplin M: Endocrine tumours of the gastrointestinal tract. Biotherapy for metastatic endocrine tumours. Best Pract Res Clin Gastroenterol 19: 617-636, 2005.

19. Oberg K: Interferon in the management of neuroendocrine GEP-tumors: A review. Digestion 62 (Suppl 1): S92-S97, 2000.
20. Dahan L, Bonnetain F, Rougier P, Raoul JL, Gamelin E, Etienne PL, Cadiot G, Mitry E, Smith D, Cvitkovic F, et al Phase III trial of chemotherapy using 5-fluorouracil and streptozocin compared with interferon alpha for advanced carcinoid tumours: FNCLCC-FFCD 97101. Endocr Relat Cancer 16: 1351-1361, 2009

21. Yao JC, Lombard-Bohas C, Baudin E, Kvols LK, Rougier P, Ruszniewski P, Hoosen S, St Peter J, Haas T, Lebwohl D, et al: Daily oral everolimus activity in patients with metastatic pancreatic neuroendocrine tumors after failure of cytotoxic chemotherapy a phase II trial. J Clin Oncol 28: 69-76, 2010.

22. Rindi G, Kloppel G, Alhman H, Caplin M, Couvelard A, de Herder WW, Erikssson B, Falchetti A, Falconi M, Komminoth P, et al: TNM staging of foregut (neuro)endocrine tumors: A consensus proposal including a grading system. Virchows Arch 449: 395-401, 2006.

23. Hentic O, Couvelrand A, Rebours V, Zappa M, Dokmak S Hammel P, Maire F, O'Toole D, Lévy P, Sauvanet A and Ruszniewski P: Ki-67 index, tumor differentiation, and extent of liver involvement are independent prognostic factors in patients with liver metastases of digestive endocrine carcinomas. Endocr Relat Cancer 18: 51-59, 2010.

24. Couverald A, Deschamps L, Ravaud P, Baron G, Sauvanet A Hentic O, Colnot N, Paradis V, Belghiti J, Bedossa P and Ruszniewski P: Heterogeneity of tumor prognostic markers: A reproducibility study applied to liver metastases of pancreatic endocrine tumors. Mod Pathol 22: 273-281, 2009.

25. Klimstra DS, Modlin IR, Adsay NV, Chetty R, Deshpande V, Gönen M, Jensen RT, Kidd M, Kulke MH, Lloyd RV, et al: Pathology reporting of neuroendocrine tumors: Application of the Delphic consensus process to the development of a minimum pathology data set. Am J Surg Pathol 34: 300-313, 2010.

26. Kulke MH, Hornick JL, Frauenhoffer C, Hooshmand S, Ryan DP, Enzinger PC, Meyerhardt JA, Clark JW, Stuart K, Fuchs CS and Redston MS: O6-methylguanine DNA methyltransferase deficiency and response to temozolomide-based therapy in patients with neuroendocrine tumors. Clin Cancer Res 15: 338-345, 2009.

27. O'Toole D, Couverlard A, Rebours V, Zappa M, Hentic O, Hammel P, Levy P, Bedossa P, Raymond E and Ruszniewski P: Molecular markers associated with response to chemotherapy in gastro-entero-pancreatic neuroendocrine tumors. Endocr Relat Cancer 17: 847-856, 2010

28. McEntee GP, Nagorney DM, Kvols LK, Moertel CG and Grant CS: Cytoreductive hepatic surgery for neuroendocrine tumors. Surgery 108: 1091-1096, 1990.

29. Moertel CG, Lavin PT and Hahn RG: Phase II trial of doxorubicin therapy for advanced islet cell carcinoma. Cancer Treat Rep 66: 1567-1569, 1982.

30. Moertel CG, Lefkopoulo M, Lipsitz S, Hahn RG and Klaassen D: Streptozocin-doxorubicin, streptozocin-fluorouracil or chlorozotocin in the treatment of advanced islet-cell carcinoma. N Eng J Med 326: 519-523, 1992

31. Eriksson B, Skogseid B, Lundqvist G, Wide L, Wilander E and Oberg K: Medical treatment and long-term survival in a prospective study of 84 patients with endocrine pancreatic tumors. Cancer 65: 1883-1890, 1990.

32. Delaunoid T, Decreux M, Boige V, Dromain C, Sabourin JC, Duvillard P, Schlumberger M, de Baere T, Rougier P, Ruffie $\mathrm{P}$, et al: The doxorubicin-streptozocin combination for the treatment of advanced well-differentiated pancreatic endocrine carcinoma; A judicious option? Eur J Cancer 40: 515-520, 2004.

33. Fjallskog ML, Janson ET, Falkmer UG, Vatn MH, Oberg KE and Eriksson BK: Treatment with combined streptozocin and liposomal doxorubicin in metastatic endocrine pancreatic tumors. Neuroendocrinology 88: 53-58, 2008

34. Bajetta E, Catena L, Procopio G, De Dosso S, Bichisao E, Ferrari L, Martinetti A, Platania M, Verzoni E, Formisano B and Bajetta R: Are capecitabine and oxaliplatin (XELOX) suitable treatments for progressing low-grade and high-grade neuroendocrine tumours? Cancer Chemother Pharmacol 59: 637-642, 2007.

35. Cassier PA, Walter T, Eymard B, Ardisson P, Perol M, Paillet C, 114 Chayvialle JA, Scoazec JY, Hervieu V and Bohas CL: 115 Gemcitabine and oxaliplatin combination chemotherapy for metastatic well-differentiated neuroendocrine carcinomas a single-center experience. Cancer 115: 3392-3399, 2009.

36. Ramanathan RK, Cnaan A, Hahn RG, Carbone PP and Haller DG: Phase II trial dacarbazine (DTIC) in advanced pancreatic islet cell carcinoma. Study of the Eastern Cooperative Oncology Group-E6282. Ann Oncol 12: 1139-1143, 2001. 
37. Vera K, Djafari L, Faivre S, Guillamo JS, Djazouli K, Osorio M, Parker F, Cioloca C, Abdulkarim B, Armand JP and Raymond E: Dose-dense regimen of temozolomide given every other week in patients with primary central nervous system tumors. Ann Oncol 15: 161-171, 2004.

38. Strosberg JR, Fine RL, Choi J, Nasir A, Coppola D, Chen DT, Helm J and Kvols L: First-line chemotherapy with capecitabine and temozolomide in patients with metastatic pancreatic endocrine carcinomas. Cancer 117: 268-275, 2011.

39. Di Bartolomeo M, Bajetta E, Bochicchio AM, Carnaghi C, Somma L, Mazzaferro V, Visini M, Gebbia V, Tumolo S and Ballatore P: A phase II trial of dacarbazine, fluorouracil and epirubicin in patients with neuroendocrine tumours. A study by the Italian Trials in Medical Oncology (I.T.M.O.) Group. Ann Oncol 6: 77-79, 1995.

40. Frame J, Kelsen D, Kemeny N, Cheng E, Niedzwiecki D Heelan R and Lippermann R: A phase II trial of streptozocin and Adriamycin in advanced APUD tumors. Am J Clin Oncol 11: 490-495, 1998

41. Sun W, Lipsitz S, Catalano P, Mailliard JA and Haller DG; Eastern Cooperative Oncology Group: Phase II/III study of doxorubicin with fluorouracil compared with streptozocin with fluorouracil or dacarbazine in the treatment of advanced carcinoid tumors: Eastern Cooperative Oncology Group Study E1281. J Clin Oncol 23: 4897-4904, 2005.

42. Eriksson B, Kloppel G, Krenning E, Ahlman H, Plöckinger U, Wiedenmann B, Arnold R, Auernhammer C, Körner M, Rindi G and Wildi S; Frascati Consensus Conference participants: Consensus guidelines for the management of patients with digestive neuroendocrine tumors-well-differentiated jejunal-ileal tumor/carcinoma. Neuroendocrinology 87: 8-19, 2008.

43. Yao JC, Phan A, Hoff PM, Chen HX, Charnsangavej C, Yeung SC, Hess K, Ng C, Abbruzzese JL and Ajani JA: Targeting vascular endothelial growth factor in advanced carcinoid tumor: A random assignment phase II study of depot octreotide with bevacizumab and pegylated interferon alpha-2b. J Clin Oncol 26: 1316-1323, 2008

44. Konno H, Arai T, Tanaka T, Baba M, Matsumoto K, Kanai T, Nakamura S, Baba S, Naito Y, Sugimura H, et al: Antitumor effect of a neutralizing antibody to vascular endothelial growth factor on liver metastasis of endocrine neoplasm. Jpn J Cancer Res 89: 933-939, 1998.

45. Zhang J, Jia Z, Li Q, Wang L, Rashid A, Zhu Z, Evans DB, Vauthey JN, Xie K and Yao JC: Elevated expression of vascular endothelial growth factor correlates with increased angiogenesis and decreased progression-free survival among patients with low-grade neuroendocrine tumors. Cancer 109: 1478-1486, 2007.

46. Kunz PL, Kuo T, Zahn JM, Kaiser HL, Norton JA, Longacre BC, Ford JM, Balise RR and Fisher GA: A phase II study of capecitabine, oxaliplatin, and bevacizumab for metastatic or unresectable neuroendocrine tumors. J Clin Oncol 28 (Suppl 15): S4104, 2010.

47. Mitry E, Walter T, Baudin E, Kurtz J-E, Ruszniewski P, Dominguez-Tinajero S, Bengrine-Lefevre L, Cadiot $G$ Dromain C, Farace F, et al: Bevacizumab plus capecitabine in patients with progressive advanced well-differentiated neuroendocrine tumors of the gastro-intestinal (GI-NETs) tract (BETTER trial) - A phase II non-randomised trial. Eur J Cancer 18: 3107-3115, 2014

48. Hobday TJ, Rubin J, Holen K, Picus J, Donehower R, Maples RM Lloyd R, Mahoney M and Erlichman C: MC044h, a phase II trial of sorafenib in patients (pts) with metastatic neuroendocrine tumors (NET) A Phase II Consortium (P2C) study. J Clin Oncol 25 (Suppl 18): S4504, 2007.
49. Phan AT, Yao JC, Fogelman DR, Hess KR, Ng CS, MalinowskiSA Regan E and Kulke M: Prospective, multi-institutional phase II study of GW786034 (pazopanib) and depot octreotide (sandostatin LAR) in advanced low-grade neuroendocrine carcinoma (LGNEC). J Clin Oncol 28 (Suppl 15): S4001, 2010.

50. Raymond E, Faivre S, Hammel P and Ruszniewski P: Sunitinib paves the way for targeted therapies in neuroendocrine tumors. Target Oncol 4: 253-254, 2009.

51. Kulke MH, Lenz HJ, Meropol NJ, Posey J, Ryan DP, Picus J, Bergsland E, Stuart K, Tye L, Huang X, et al: Activity of sunitinib in patients with advanced neuroendocrine tumors. J Clin Oncol 26: 3403-3410, 2008.

52. Raymond E, Dahan L, Raoul JL, Bang YJ, Borbath I, Lombard-Bohas C, Valle J, Metrakos P, Smith D, Vinik A, et al: Sunitinib malate for the treatment of pancreatic neuroendocrine tumors. N Eng J Med 364: 501-513, 2011.

53. Moreno A, Akcakanat A, Munsell MF, Soni A, Yao JC and Meric-Bernstam F: Antitumor activity of rapamycin and octreotide as single agents or in combination in neuroendocrine tumors. Endocr Relat Cancer 15: 257-266, 2008.

54. Yao JC, Phan AT, Chang DZ, Wolff RA, Hess K, Gupta S, Jacobs C, Mares JE, Landgraf AN, Rashid A and Meric-Bernstam F: Efficacy of RAD001 (everolimus) and octreotide LAR in advanced low- to intermediate-grade neuroendocrine tumors: Results od a phase II study. J Clin Oncol 26: 4311-4318, 2008

55. O'Reilly KE, Rojo F, She QB, Solit D, Mills GB, Smith D, Lane H, Hofmann F, Hicklin DJ, Ludwig DL, et al: MTor inhibition induces upstream receptor tyrosine kinase signalling and activates Akt. Cancer Res 66: 1500-1508, 2006.

56. Yao JC, Shah MM, Ito T, Bohas CL, Wolin EM, Van Cutsem E, Hobday TJ, Okusaka T, Capdevila J, de Vries EG, et al: Everolimus for advanced pancreatic neuroendocrine tumors. N Eng J Med 364: 514-523, 2011

57. Yao JC, Hainsworth JD, Baudin E, Peeters M, Hoersch D, Klimovsky LB, Grouss K, Jehl V and Pavel M: Everolimus plus octreotide LAR $(\mathrm{E}+\mathrm{O})$ versus placebo plus octreotide LAR $(\mathrm{P}+\mathrm{O})$ in patients with advanced neuroendocrine tumors (NET): Updated results of a randomized, double-blind, placebo-controlled, multicentre phase III trial (RADIANT-2). J Clin Oncol 29 (Suppl 4): S159, 2011

58. Kos-KudłaB,Blicharz-DorniakJ,StrzelczykJ,Bałdys-WaligórskaA, Bednarczuk T, Bolanowski M, Boratyn-Nowicka A, Borowska M, Cichocki A, Ćwikła JB, et al: Diagnostic and therapeutic guidelines for gastro-entero-pancreatic neuroendocrine neoplasms (recommended by the Polish Network of Neuroendocrine Tumours). Endokrynol Pol 68: 79-110, 2017.

59. Mitry E, Baudin E, Ducreux M, Sabourin JC, Rufié P, Aparicio T, Aparicio T, Lasser P, Elias D, Duvillard P, et al: Treatment of poorly differentiated neuroendocrine tumours with etoposide and cisplatin. Br J Cancer 81: 1351-1355, 1999.

60. Seitz J, Perrier H, Giovannini M, Monges G, Fourdan O, Barrière $\mathrm{N}$ and Viens $\mathrm{P}$ : Cancers neuroendocrines anaplasiques avances interet de l'association VP16-CDDP. Bull Cancer 82: 433-434, 1995

61. Mitry E and Rougier P: The treatment of undifferentiated neuro- 102 endocrine tumors. Crit Rev Oncol Hematol 37: 47-51, 2001. 